\title{
ON POSSIBLE DETERMINATION OF THE SPEED OF THE GRAVITY SIGNAL IN SPACE WITH HELP OF GRADIOMETRY
}

\author{
Janusz B. Zieliński ${ }^{(1)}$, Robert R.Gałązka ${ }^{(2)}$, Roberto Peron ${ }^{(3)}$ \\ (1) Space Research Centre, Polish Academy of Sciences, \\ ul. Bartycka 18, 00-716 Warsaw, POLAND \\ jbz@.cbk.waw.pl \\ (2) Institute of Physics, Polish Academy of Sciences, \\ Al.Lotników 32/46, 02-668 Warsaw, POLAND \\ galaz@ifpan.edu.pl \\ 3/ Istituto di Fisica dello Spazio Interplanetario, Istituto Nazionale di Astrofisica, \\ Via del Fosso del Cavaliere, 00133 Roma, ITALY \\ roberto.peron@ifsi-roma.inaf.it
}

\section{INTRODUCTION}

Currently two well established physical theories describe the fundamental interactions: quantum field theory - Standard Model and theory of gravity - General Theory of Relativity. Many theoretical works are conducted to combine these two theories to one "Theory of Everything". Concerning the gravity, experimental data are in agreement with Einstein theory of gravity, from the Mercury perturbation motion to time corrections of GPS satellites clocks. However, a number of questions still remains open. Gravity waves and gravitons are, till now, not observed. Another question is how fast the curvature of spacetime can change. Our proposal of the experiment can contribute to the discussion of these problems.

\section{SATELLITE GRADIOMETRY}

Space technology provided the mankind with new possibilities and tools for the new frontiers in experimental science. One of these is the Global Navigation Satellite System; the particular realization of GNSS - Galileo - is being the main topic of this conference. Another one is the gradiometry, the modern technology which allows the measurement of the gravity field variations as function of position as well as time. The best example of the application of this technology is the project GOCE that is going to be launched in few months from now. Theoretical foundations for this method were developed by R. Rummel [1] and other geodesists. The gradiometer applied for this mission is probably the top achievement of the space technology. The sensitivity of this instrument is of the order of $10^{-3} \mathrm{EU}$, where EU denotes the Eötvös unit of the gravity acceleration gradient: $1 \mathrm{EU}=10^{-9} \mathrm{~m} \mathrm{~s}^{-2} / \mathrm{m}$. The gradiometer consists of three pairs of accelerometers, mounted on three perpendicular axes, with the separation between accelerometers in each pair equal to $0.5 \mathrm{~m}$. The accelerometer noise is smaller than $2 \times 10^{-13} \mathrm{~m} \mathrm{~s}^{-2} \mathrm{~Hz}^{-1 / 2}$ in the defined measurement bandwidth (from 0.005 to $0.1 \mathrm{~Hz}$ ) [2]. The orbit of the GOCE satellite is planned to have the following parameters: mean altitude $\approx 250 \mathrm{~km}, i=96.5^{\circ}$. At this altitude the variations in the vertical gradient $T_{r r}$, 
along the orbit, are in the range of \pm 1 EU. So, the sensitivity of the GOCE gradiometer is much higher than the amplitude of the measured signal.

Expressions for the gradient are given by Łatka [3] or in the ERS report by Johannessen and Aguirre-Martinez [4]. We will discuss here the vertical component $T_{r r}$ only of the gradient tensor, because this component is the most convenient for the measurements. This expression after Łatka is

$$
T_{r r}=\frac{1}{r^{2}} \sum_{n=2}^{N}(n+1)(n+2)\left(\frac{a_{E}}{r}\right)^{n+1} \frac{G M}{a_{E}} \sum_{m=0}^{n}\left(\bar{C}_{n m} \cos m \lambda+\bar{S}_{n m} \sin m \lambda\right) \bar{P}_{n m}(\sin \varphi)
$$

and after ESA Report

$$
V_{r r}=\frac{G M}{a_{E}} \sum_{n=0}^{N} \frac{(n+1)(n+2)}{a_{E}{ }^{2}}\left(\frac{a_{E}}{r}\right)^{n+3} \sum_{m=-n}^{n} \sum_{k=-n}^{n} K_{n m} F_{n m k}(I) \exp [i(k u+m \Lambda)]
$$

These two expressions are equivalent, but $T_{r r}$ denotes the gradient in the perturbing gravity potential, without the zero and second order terms, while $V_{r r}$ is the gradient in the whole Earth's potential.

A well-known feature of the gravity potential field is the attenuation of the signal with altitude. This is the main reason why the missions like GOCE must be realized with an orbit as low as possible. In the case of the gravity acceleration gradient, for the $r r$ component the attenuation factor is equal to

$$
\text { att. } f a c=\frac{(n+1)(n+2)}{a_{E}{ }^{2}}\left(\frac{a_{E}}{r}\right)^{n+3}
$$

where $n$ is the degree of the spherical harmonics expansion, $a_{E}$ is the radius of the Earth sphere and $r$ is the distance from the Earth center.

The attenuation has two features:

- the total value of the function (1) is decreasing with the altitude above the Earth surface

- the resolution is decreasing because of the more rapid decline of the higher order terms of the series (1).

Consequently, the higher the orbit of the satellite with gradiometer the more global (low frequency) the gravitational gradient signal is.

Some numerical calculations can give us an idea about the evolution of the gravitational gradient with orbital height. 


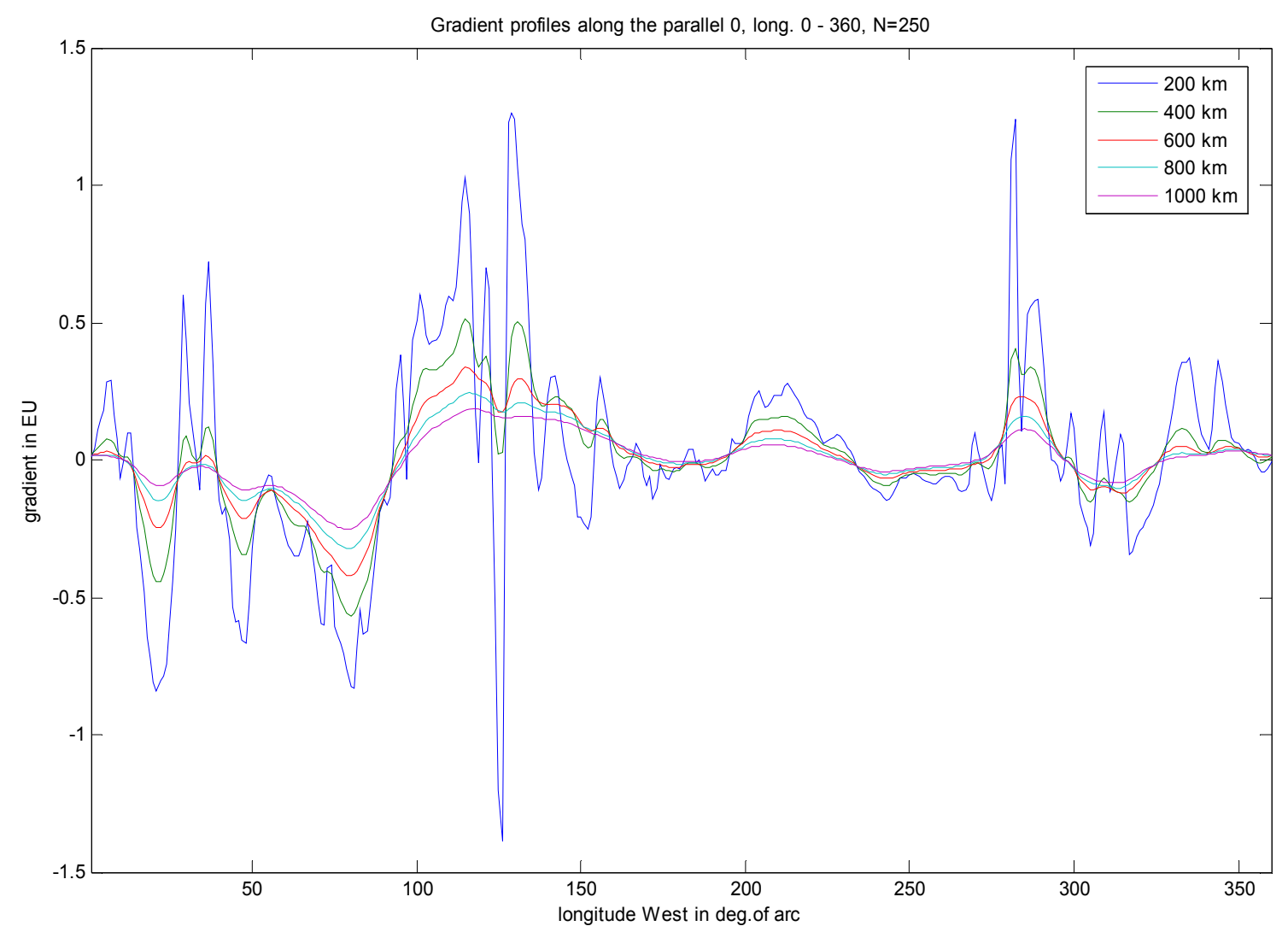

Fig 1. Gradient profiles in the equator plane for orbital heights $200 \mathrm{~km}-1000 \mathrm{~km}$

Fig. 1 shows the variation of the function (1) along the equator on the altitude range 200 $\mathrm{km}$ to $1000 \mathrm{~km}$, taking the EGM96 gravity field model limited to $n=250$. We can see that at the altitude ca. $200 \mathrm{~km}$ the gradient has very strong and high frequency variations in the range of \pm 1 EU. These variations are attenuated with the rise of the height of the orbit because the higher order terms are vanishing.

Figures $2-5$ present the evolution of the $T_{r r}$ with height. The reader should note the change of the vertical scale on the consecutive diagrams. The diagrams Fig. 4 and Fig. 6 show the gradient functions for the height range of satellites GPS and Galileo. We can see that at this height we have the signal with frequency $1 / \pi$ and amplitude of the order of $10^{-4} \mathrm{EU}$. The Fig. 5 demonstrates the evolution above the distance of the geostationary satellites 


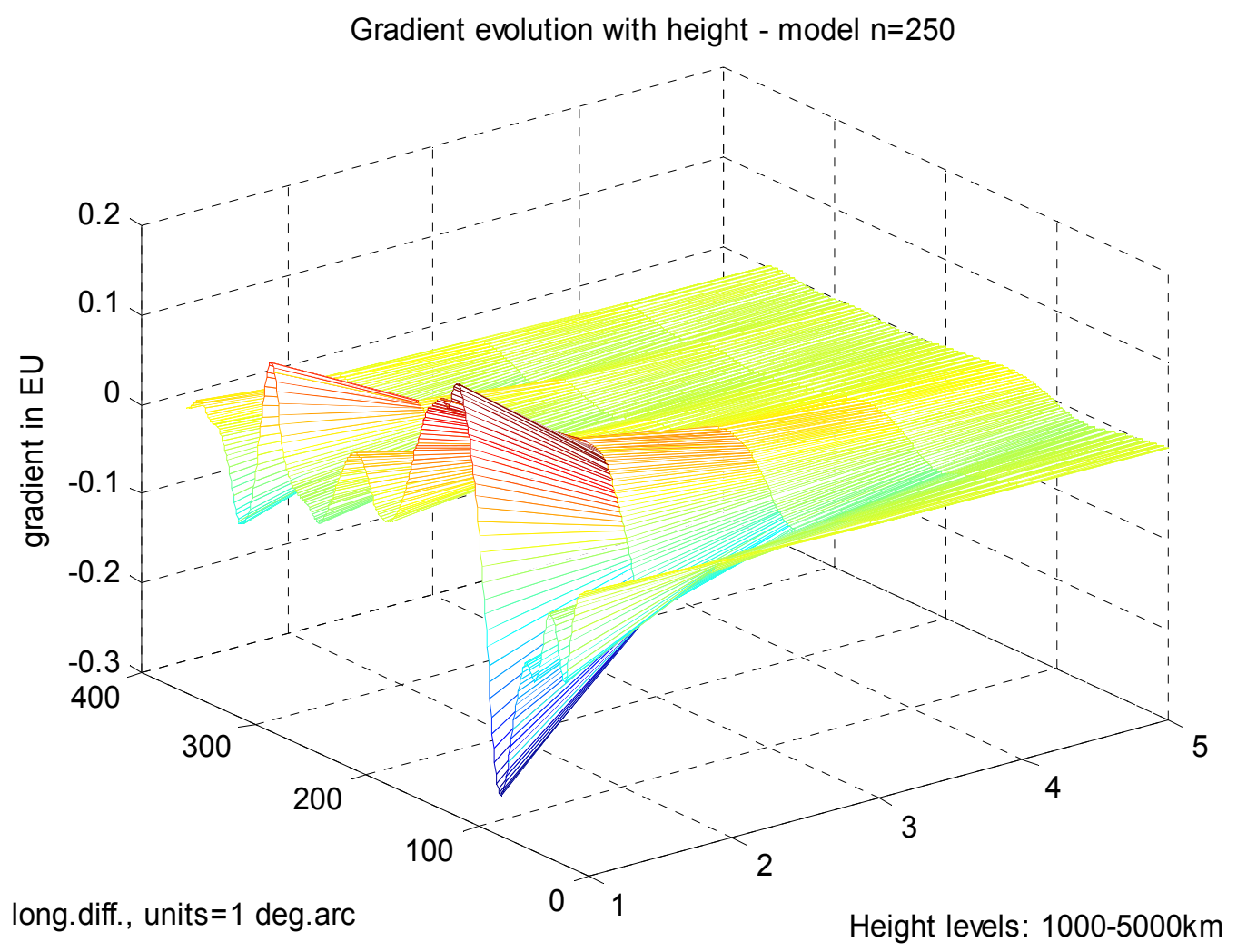

Fig. 2. Evolution of the gradient for the orbital heights $1000 \mathrm{~km}-5000 \mathrm{~km}$

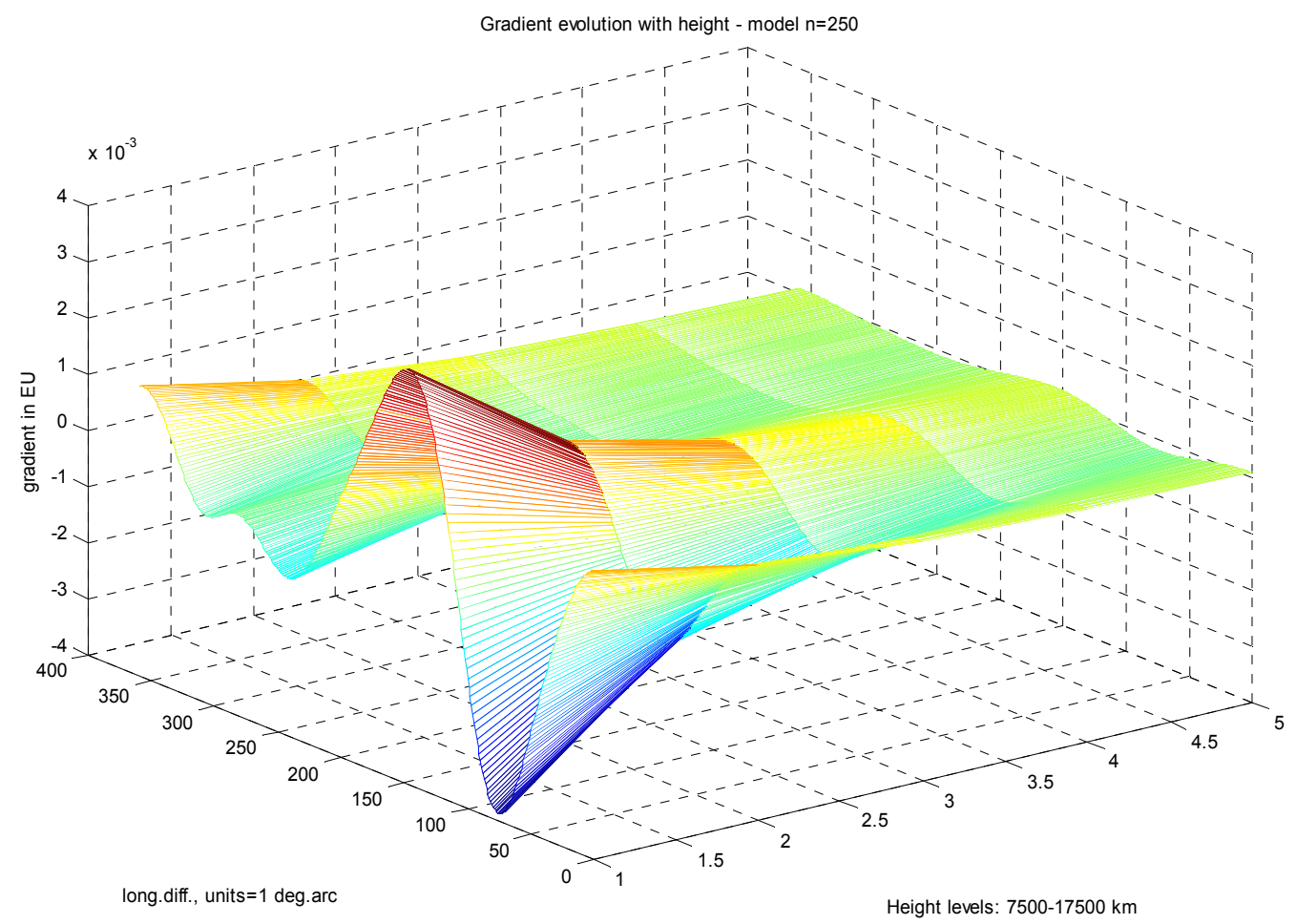

Fig. 3. Evolution of the gradient for the orbital heights $7500 \mathrm{~km}-17500 \mathrm{~km}$ 


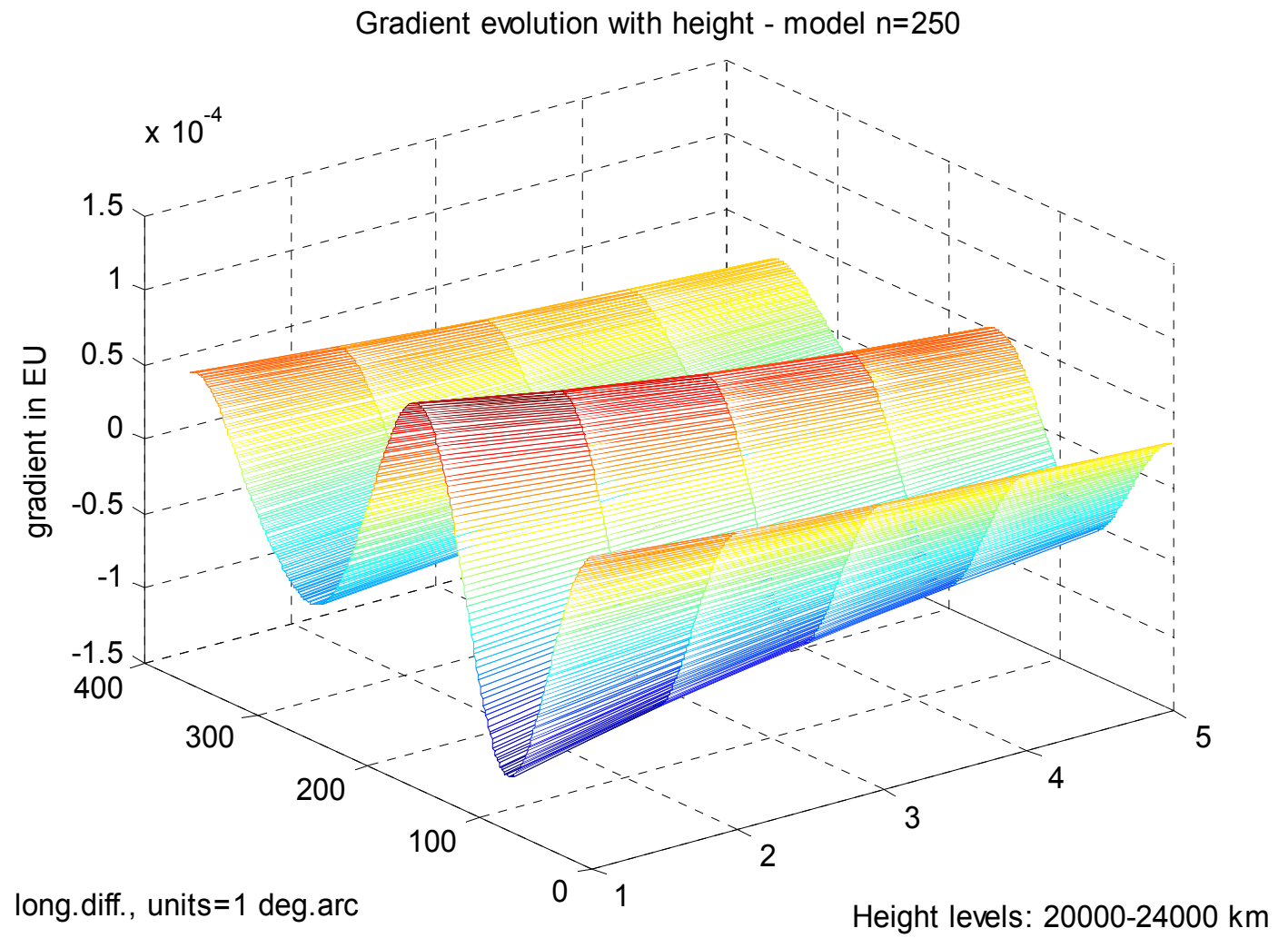

Fig.4. Evolution of the gradient for the orbital heights $20000 \mathrm{~km}-24000$

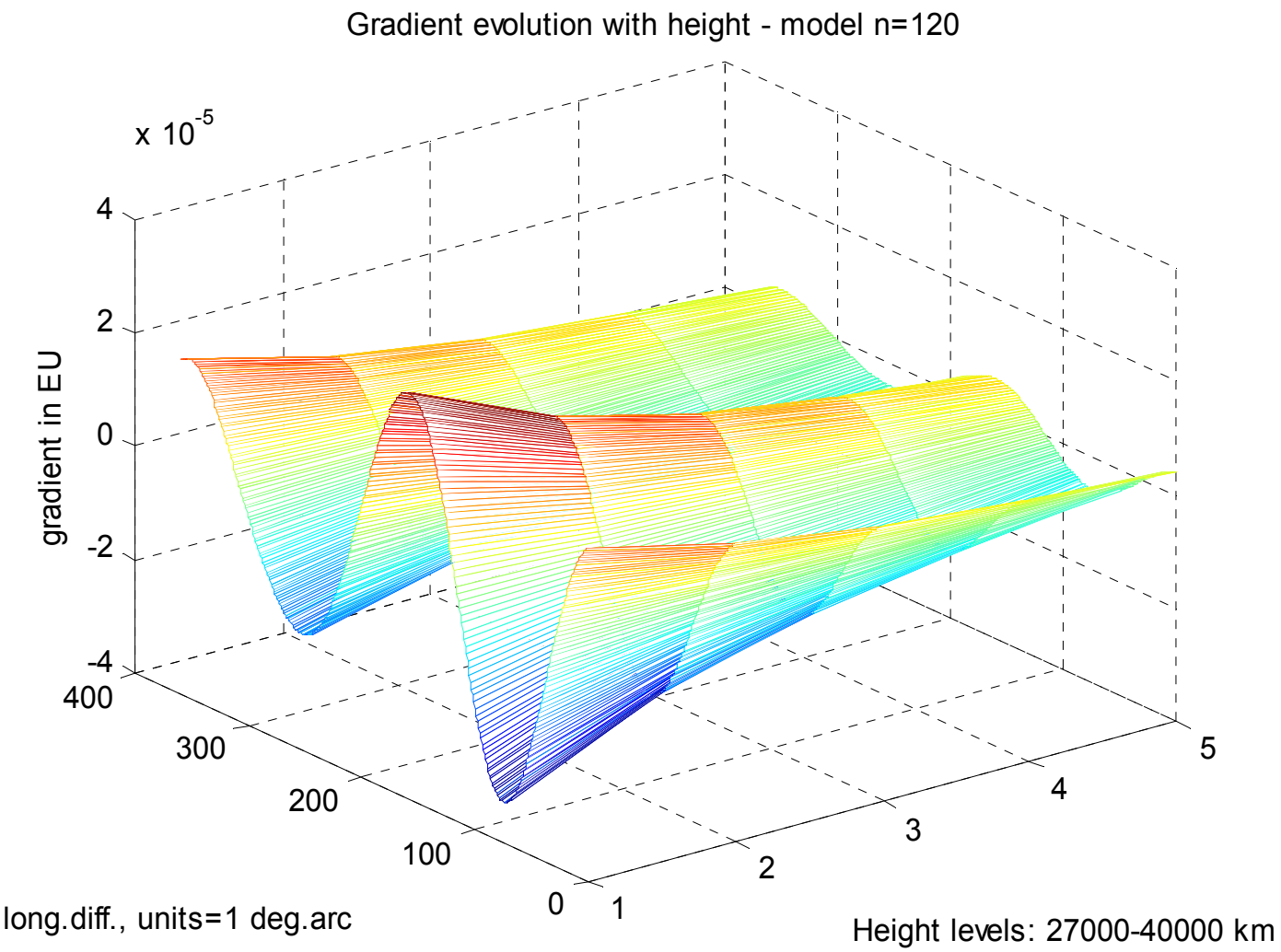

Fig. 5. Gradient evolution for the orbital heights $27000 \mathrm{~km}-40000 \mathrm{~km}$ 
Expressions (1) or (2) can be also applied for the Upward Continuation (UC) Procedure [5]. By Upward Continuation we understand the mathematical operation when the value of the function is known on the certain level and we are going to calculate it on the higher level. Because the (absolute) value of the geopotential is decreasing with the geocentric distance, and the same is true with functionals like gravity acceleration and gradients, UC procedure is stable and can be safely applied, assuming that the gravity field model is accurate enough.

\section{CONCEPT FOR DETERMINATION OF THE VELOCITY OF THE GRAVITATIONAL SIGNAL}

Now, let us consider the possibility of application of the gradiometry for the determination of the velocity of propagation of the gravitational signal in space. First, let us consider the theoretical possibility of such an experiment then let us discuss the constraints related to the accuracy of the solution. Let us ponder if the GNSS satellites provide a convenient tool for the realization of this experiment.

In the beautiful book on gravity Bernard Schutz said: The only part of the gravitational field of a wave that we can measure directly is the non-uniform part, which acts in such way that one section of an apparatus is affected by gravity differently than another. We can therefore only register the differences in gravitational acceleration across the region occupied by our experiment [6]. This is exactly the principle of the gradiometer.

We consider the Earth-centered inertial reference (ECIR) frame oriented to distant stars but centered in the Earth's center of mass. The Earth is rotating with respect to this frame together with its gravity field. On the other hand the GNSS satellites are orbiting in the same frame and their circular orbits approximately form the sphere that is stable in ECIR frame. The rotating undulated Earth gravity field is crosscut by the sphere and the gravitational potential on this sphere is time dependent, as well as its gradients. If the satellites are equipped with gradiometers the variable gradient can be measured.

The radius of the sphere depends on the altitude of the GNSS orbits. The altitudes of the GPS constellation and the Galileo foreseen one are $20183 \mathrm{~km}$ and $23222 \mathrm{~km}$, respectively. This is about $3000 \mathrm{~km}$ difference in height. For the velocity of light it is ca. $0.01 \mathrm{~s}$. We can also imagine a substantially higher constellation with the height difference of even $30000 \mathrm{~km}$ that will produce $0.1 \mathrm{~s}$ of the time difference. 


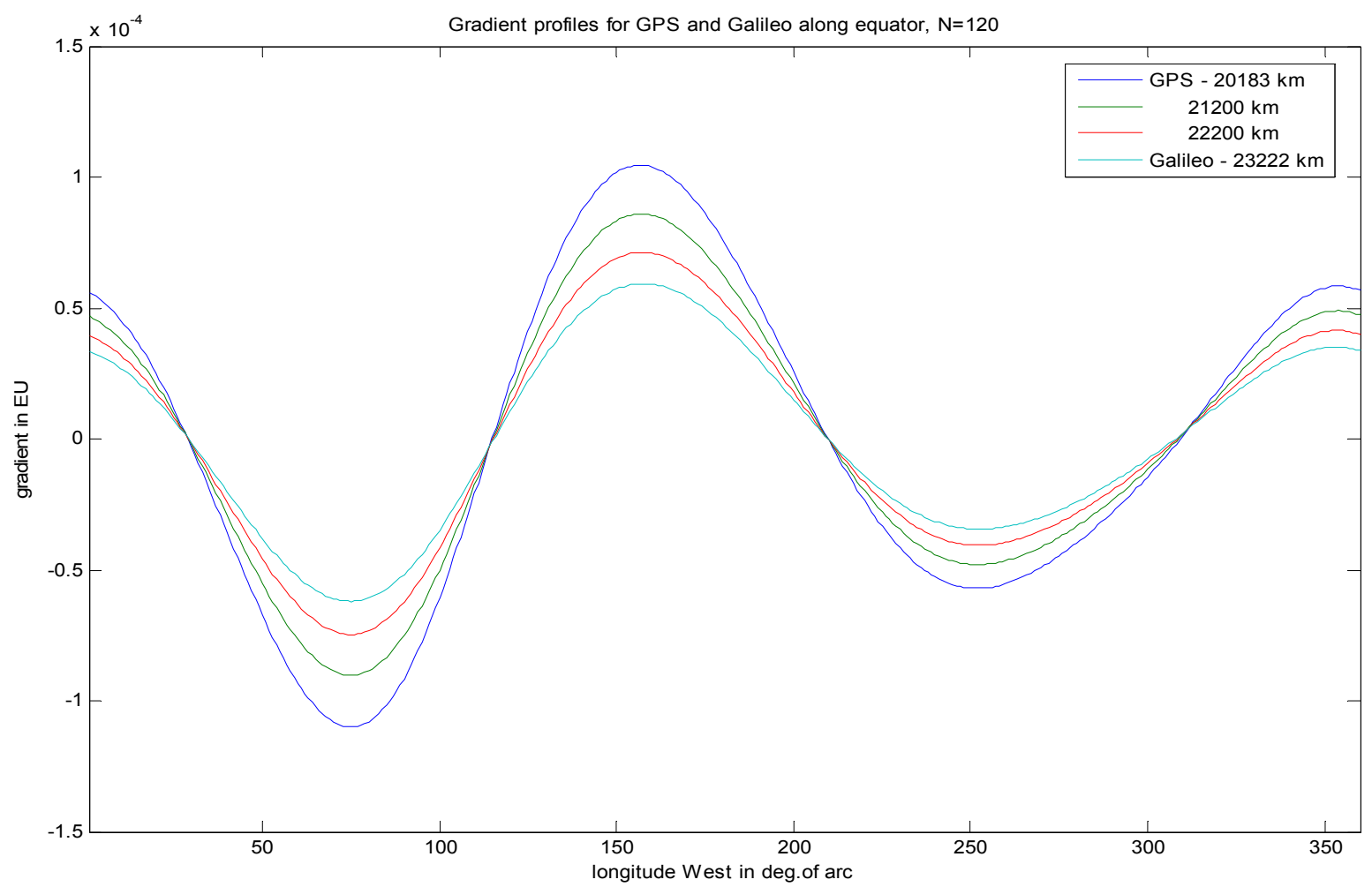

Fig. 6. Gradient profiles for orbital heights of GPS and Galileo

Now, let us disregard the Newtonian notion of the gravitational potential rigidly connected with the generating mass and let us think in terms of the general relativistic concept of gravity. Then, the same gravitational gradient signal should arrive to the Galileo sphere $0.01 \mathrm{~s}$ later than to the GPS sphere. If we could measure this time interval accurately enough we could determine the speed of propagation of the gravitational signal.

We can consider the Earth's fixed coordinates $\varphi$ and $\lambda$ rotating with respect to the ECIR celestial coordinates $\delta$ and $\alpha$. If we take the intersection on GPS sphere of a given arbitrary celestial meridian $\alpha_{0}$ with the equatorial plane $\varphi=\delta=0$ then the value of the gravitational gradient at this point $\mathrm{P}_{0}$ will be a function of time. We will have the same along the straight line going upwards from $\mathrm{P}_{0}$ but with constant orientation in the celestial frame. The value of $T_{r r}$ along this line will vary as function of time and geocentric distance $r$. This is shown schematically in Fig. 7a and $7 \mathrm{~b}$.

Given the instant $t_{i}$ and the distance $r_{i}$, let us denote the gradient with $T_{r r}\left(t_{i}, r_{i}\right)$; for the same instant $t_{i}$ but another distance $r_{k}$ we have $T_{r r}\left(t_{i}, r_{k}\right)$. In the Newtonian definition of the gravity field where the field is rigidly connected to the generating mass the value $T_{r r}$ for the same instant $t_{i}$ but different radius $r_{k}$ will differ by the upward continuation operator depending on height above the Earth.

Let us write

$$
T_{r r}\left(t_{1}, r_{2}\right)=T_{r r}\left(t_{1}, r_{l}\right) \otimes U C
$$

where the symbol $\otimes U C$ denotes application of the Upward Continuation Procedure. 
However, in general relativity we assume that the gravity is propagating in space with the velocity $c_{g}$. The gravitational signal is traveling from the height $r_{1}$ to the height $r_{2}$ during the time interval

$$
\Delta t=\frac{r_{2}-r_{1}}{c_{g}}
$$
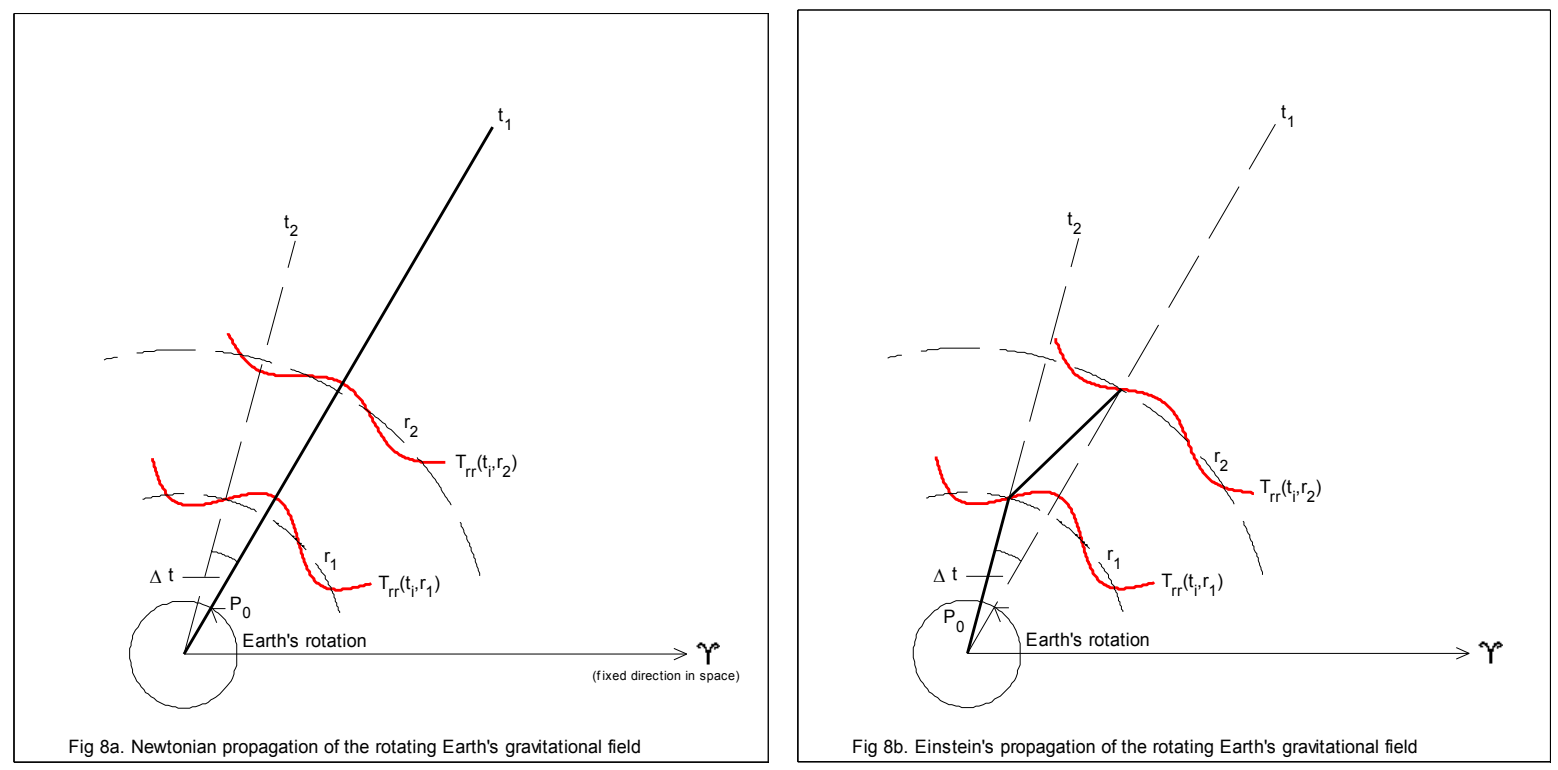

Fig.7. Earth rotation and gravity

During this time interval the Earth will rotate by the small angle $\Delta t$ as well. Because of this time delay and small rotation the gradient registered at $t_{1}$ on the level $r_{2}$ will be not the simple Upward Continuation from the $r_{l}$ at the moment $t_{l}$ but will be equal (see Fig. 8)

$$
T_{r r}\left(t_{1}, r_{2}\right)=T_{r r}\left(t_{2}, r_{1}\right) \otimes U C-\frac{d \Delta T_{r r}}{d t} \Delta t
$$

where $\frac{d \Delta T_{r r}}{d t}$ is the rate of change of the

$$
\Delta T_{r r}=T_{r r}\left(t_{i}, r_{2}\right)-T_{r r}\left(t_{i}, r_{1}\right)
$$

If we denote the last term of (6) as $\delta T_{r r}$ we get

$$
\delta T_{r r}=T_{r r}\left(t_{2}, r_{1}\right) \otimes U C-T_{r r}\left(t_{1}, r_{2}\right)
$$

and taking into account (5)

$$
c_{g}=\frac{\left(r_{2}-r_{1}\right)}{\delta T_{r r}} \cdot \frac{d \Delta T_{r r}}{d t}
$$

In this expression $c_{g}$ is considered unknown, the values $r_{1}, r_{2}$ are known from the satellite geodesy positioning methods, the rate $\frac{d \Delta T_{r r}}{d t}$ could be determined from the geopotential model, only the value $\delta T_{r r}$ must be determined by gradiometry. The geopotential model at 
present is always Newtonian and for it $\delta T_{r r}=0$ but if we measure it and find that it is $\neq 0$ then we can try to determine $c_{g}$.

Expression (9) can be considered as an observation equation because it can be written for each observation. However, we have to admit, the single measurement will give very uncertain result because the $\delta T_{r r}$ in the denominator is very small.

Let us look to this problem from another side. The $T_{r r}$ gradient curves projected on the circles with radii $r_{1}$ and $r_{2}$ can be treated as a harmonic signals described by the time series as function of the Earth's rotation, for which we can determine the phase and the amplitude of several terms in particular the zero term. The amplitudes for the upper curve will be related to the lower one by the Upward Continuation. But the phase of the upper one will be shifted with respect to the lower one by the angle - or time difference -

$$
\Delta t=\frac{r_{2}-r_{1}}{c_{g}}
$$

creating the shifted twin signal, let call it the "shadow function" (see Fig. 8).

The solution of the problem will consist on the determination by gradient measurements of the phase difference of both signals - upper and lower.

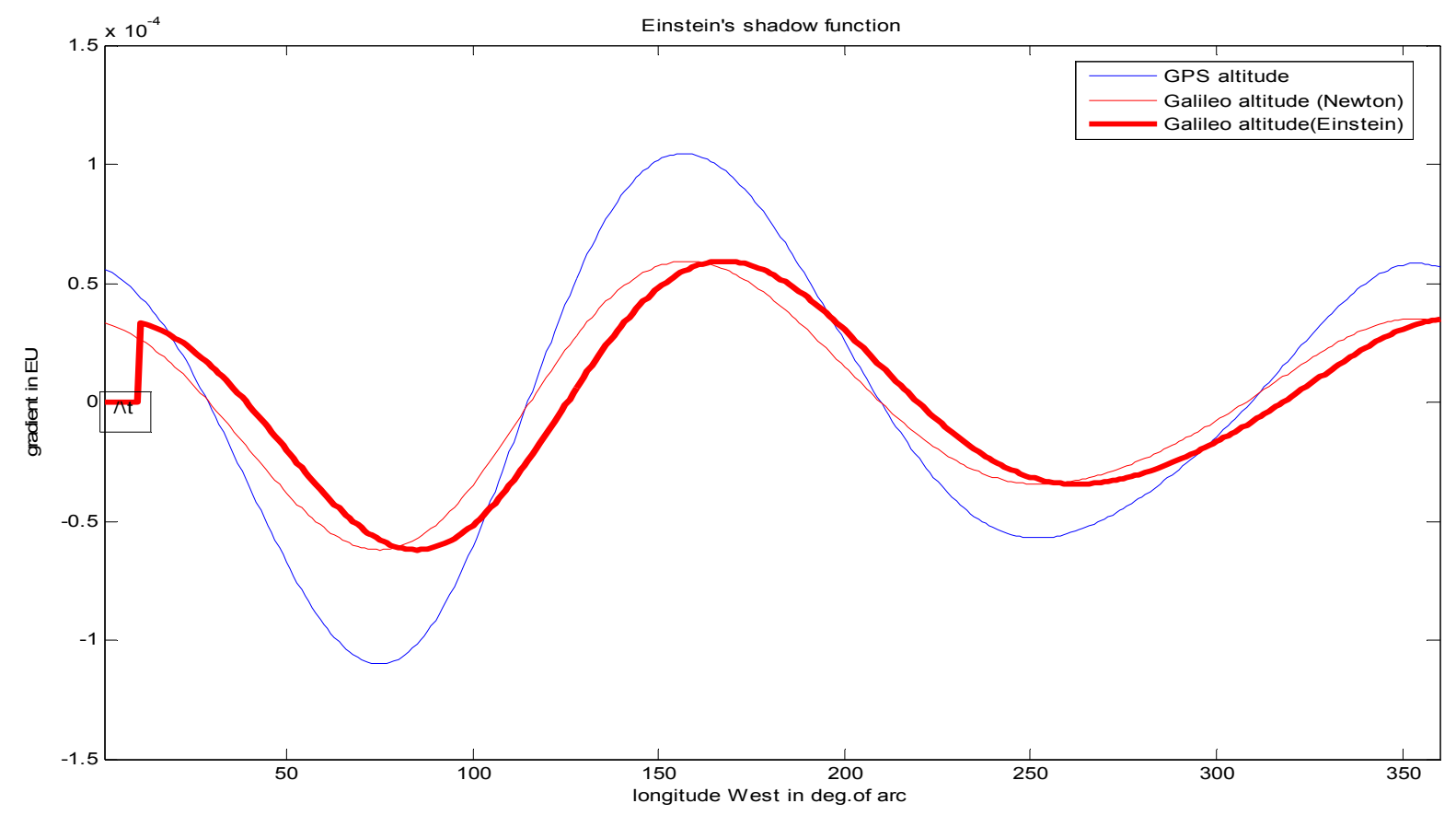

Fig. 8. The shadow function for Galileo orbital height

For the case of the constellations GPS and Galileo the difference of the height of the orbits is roughly $3000 \mathrm{~km}$. This is equivalent to $\Delta t=0.01 \mathrm{~s}$ and it is in turn equivalent to $0 . " 15$ of the angle of rotation of the Earth. On the surface of the Earth it gives ca. $4.5 \mathrm{~m}$ but on the orbital height of Galileo it will be ca. $18 \mathrm{~m}$.

\section{DISCUSSION OF ACCURACY}

Now we can discuss the accuracy of the solution to this problem.

It seems that there are two principal constraints to the realization of this experiment: precision of the gradient measurement and precision of the time determination. Let us discuss them separately. 
The GOCE gradiometer is nowadays probably the most advanced instrument of this type. However some works are conducted in different countries which show that we are not yet at the limit of the possible measurement precision. In the Istituto di Fisica dello Spazio Interplanetario in Roma very successful experiments by Iafolla with the Italian Spring Accelerometer (ISA) open new possibilities for further improvement in accuracy and performance of a new generation gradiometer[7]. In the US the group of Paik published some results hoping to achieve the accuracy $10^{-5} \mathrm{EU}[8]$.

In the project STEP (Satellite Test of the Equivalence Principle) the accelerometer prepared for this mission has a sensitivity of $10^{-18} \mathrm{~g}$ which is equivalent to $10^{-17} \mathrm{~m} \mathrm{~s}^{-2}$. The gradiometer consisting of the pair of such accelerometers should have a sensitivity of $10^{-8} \mathrm{EU}$ [9]. Of course, building a gradiometer is not simply putting two accelerometers one aside the other. It would be so if you could align them with the required accuracy, and maintain this for all the measurement period. This is not easy, nevertheless is not hopeless.

$\underline{\text { Tab. } 1}$

Expected development in gradiometry

1. GOCE 10-3 EU

2. IFSI 10-4 EU

3. Paik 10-5 EU

4. STEP 10-8 EU

As concerning the precision of timing in GNSS systems it is better than $10^{-8} \mathrm{~s}$. Synchronization of two different systems like Galileo and GPS could present some problem, nevertheless we are rather on the safe side.

The challenge will be rather the detection of the incoming signal, because it will be very flat. From the Fig. 6 or 8 we can see the signal shape distributed over 24 hours: the period is equal to about 12 hours and the amplitude to $1 \cdot 10^{-4} \mathrm{EU}$. It means that from the bottom to the peak of the signal we have about 6 hours. With the linear approximation we can tell that for 1 $\mathrm{s}$ we get a $0.5 \cdot 10^{-8} \mathrm{EU}$ change for the gradient. If we are interested in the $\pm 0.001 \mathrm{~s}$ accuracy in the determination of the signal arrival time it means that equivalent accuracy in the gradient measurement should be $\pm 0.5^{*} 10^{-11} \mathrm{EU}$. This level of the gradiometer precision is hardly imaginable even with very developed and sophisticated technology.

Probably the only possibility to determine the difference in the time of arrival on two different heights will be filtering technique of both curves. The shape of the curve could be modeled with sufficient accuracy using the known gravity field model. We can take into account that GNSS constellations are long living systems. Then, the statistics can be helpful.

If we have the accurate theoretical model of the curve that should be fitted by measurements then only one term of the zero order has to be determined. The accuracy of this term is roughly described as

$$
M_{0}= \pm \frac{\sigma_{0}}{\sqrt{n}}
$$

where $\sigma_{0}$ is the standard deviation of the measurement and $n$ is the number of measurements. Supposing that the measurement is done with the frequency $1 \mathrm{~Hz}$, during 24 hours we have 86400 measurements and during 12 days more than one million. With the individual measurement error $\pm 10^{-8} \mathrm{EU}$ and 12 days measurement interval we can get close to the desired accuracy $\pm 10^{-11}$. 
Unfortunately, experts in accelerometry technology are of the opinion that accuracy $\pm 10^{-8}$ EU is not achievable, also in the future [10]. Therefore, less demanding requirements for the measurement accuracy must be accepted but compensated by the increasing number of observations. Considering the continuous operation of GPS and Galileo and the number of satellites which could be available, the amount of data collected could be enormous.

\section{CONCLUSIONS}

It seems that the concept for the determination of the velocity of the gravitational signal, using the rotating Earth as the signal generator, and GNNS plus gradiometry as detector, is realistic, but of course not easy. It should provide the motivation for the development of the gradiometry technology and could widen the spectrum of scientific applications of GNSS. Also, some other satellite constellation could be considered, with lower orbits but greater height difference. Optimal combination of the gradiometry resolution and orbital height could be find with further analytical and numerical research.

\section{ACKNOWLEDGEMENT}

This paper is an extended and revised version of the presentation delivered to the $1 s t$ Colloquium on Scientific and Fundamental Aspects of the Galileo Programme, Toulouse, 1-4 October 2007 [11]. Authors wish to express the gratitude to Dr. Valerio Iafolla for the discussion and useful comments on the paper.

\section{REFERENCES}

[1] Rummel, R., F. Sanso, M. Van Gelderen, M. Brovelli, R. Koop, F. Migliaccio, E.J.O. Schrama, F. Sacredote Spherical harmonics analysis of satellite gradiometry. Neth. Geodetic Commission, No.39, 1993.

[2] Drinkwater M.R., R. Haagmans, D. Muzi, A. Popescu, R. Floberghagen, M. Kern and M. Fehringer The GOCE gravity mission: esa's first core earth explorer Proceedings of the $3^{\text {rd }}$ International GOCE User Workshop, 6-8 November, 2006, Frascati, Italy, ESA Special Publication, SP-627, ISBN 92-9092-938-3, pp.1-8, 2007.

[3] Łatka, J.K., The use of satellite gradiometry for determination of gravity anomaly. Beitraege zur Astronomischen Geodaesie und Geodynamik. Inst. Fuer Physikalische Geodaesie der Technischen Hochschule Darmstadt, 1978.

[4] Johanessen J.A., M. Aguirre-Martinez, Gravity field and steady-state ocean circulation. The Four Candidate Earth Explorer Core Mission, ESA SP-1233(1), 1999

[5] Zieliński J.B., J.K. Łatka, M. Kuźmicz-Cieślak Upward continuation of gravity gradients for validation of the GOCE measurements. Artificial Satellites-Journal of Planetary Geodesy, Vol.40, pp.219-249, 2005

[6] Schutz B., Gravity from the ground up. Cambridge University Press, 2005

[7] Iafolla V., A. Mandiello, S. Nozzoli, The High Sensitivity Italian Spring Accelerometer (ISA) for Fundamental Physics in Space. Adv. Space Res. Vol.25, No 6, pp.1241-44, 2000.

[8] Paik, H.J., B. Mashhoon, C.M. Will, Detectionof gravitomagnetic field using an orbiting superconducting gravity gradiometer. Inernet. Symp. On Experimental Gravitational Physics, Guangzhou, China, August 1987,ed. P.F.Michelson (world Scientific, Singapore), pp.229-244, 1987. 
[9] T.J. Sumner, J. Anderson, J.-P. Blaser, A.M. Cruise, T. Damour, H. Dittus, C.W.F. Everitt, B. Foulon, Y. Jafry, B.J. Kent, N. Lockerbie, F. Loeffler, G. Mann, J. Mester, C. Pegrum, R. Reinhardt, M. Sandford, A. Scheicher, C.C. Speake, R. Torii, et al. STEP(satellite test of the equivalence principle). Adv. Space Res. Vol. 39, No.2, pp.254258, 2007.

[10] Iafolla V., private communication, 2007.

[11] Zielinski, J.B.; Galazka, R.R.; Peron, R. Possible detection of the speed of the gravity signal with help of gradiometers on the GNSS orbit and higher. Proc.of 1st Colloquium on Scientific and Fundamental Aspects of the Galileo Programme, Toulouse, 1-4 October 2007, ed. Congrex, ESA's Professional Conference Organizer, 16 February 2008, CD-ROM.

Received: 2008-02-05,

Reviewed: 2008-03-18, by V. Iafolla,

Accepted: 2008-03-31. 